\title{
Application of Precession Electron Diffraction in Deformation Studies of Advanced Non-Ferrous Structural Alloys
}

\author{
Iman Ghamarian $^{1}$, Yue Liu ${ }^{1,2}$ and Peter C. Collins ${ }^{1,2}$ \\ 1. Department of Materials Science and Engineering and the Center for Advanced Research and Testing, \\ University of North Texas, Denton, TX 76203, USA \\ 2. Center for Advanced Non-Ferrous Structural Alloys (CANFSA), an NSF I/UCRC between the \\ University of North Texas (Denton, TX 76203, USA) and the Colorado School of Mines (Golden, CO \\ 8-401, USA)
}

It is well-understood that the microstructure of a material, including the defect structures present (e.g., dislocations, grain boundaries) significantly influence the mechanical properties of structural metallic materials. While the evolution of the phases in the microstructure is reasonably well-understood, and may be placed upon a thermodynamic framework, the evolution of the defect structure is less well understood. For the latter, often the research has focused on qualitative descriptions of the dislocation level details of the defect structure. This has been due to the manual analysis that has typically been required to analyze defect structures.

However, recently, the development of precession electron diffraction (PED) [1], a TEM-based tool, offers to provide new means to automatically and quantitatively assess the materials microstructure. It is possible to conduct orientation and phase mapping in the TEM using state-of-the-art precession-assisted electron diffraction orientation microscopy with high spatial resolution $(2 \mathrm{~nm})$ [2] and considerable angular resolution $\left(\sim 0.8^{\circ}\right)$ [3], which has paved the way for quantitative approaches to study challenging problems like severely deformed materials. It should be noted that the relatively low angular resolution of PED in comparison with high resolution EBSD is due to the less sensitivity of spot diffraction patterns to small lattice distortions in comparison with Kikuchi patterns.

This work focuses on the procedure of deriving dislocation density distribution map from the orientation data set of a commercially pure ultrafine grained titanium alloy acquired by PED. Initially, the angular resolution of PED is improved from $0.8^{\circ}$ to $0.4^{\circ}$ via applying Kuwahara filter. Different parameters, such as sub-region size and number of iterations, which affect the performance of the Kuwahara filter were studied extensively. An example of the capability of Kuwahara filter to improve the angular resolution is shown in Fig. 1(a-b). A simulated orientation database with cells having $0.6^{\circ}$ disorientation with its neighbors and maximum global misorientation of $6^{\circ}$ is shown in Fig. (1a). Each pixel in this plot has $0.8^{\circ}$ noise. Applying Kuwahara filter with sub-region size of five for three iterations significantly resolves the boundaries between the adjacent cells and removes noise, Fig (1b).

An index map (called image quality in EBSD) of an ultrafine grained commercially pure titanium alloy is shown in Fig. (2-a). This map reveals that the size of grains varies from $\sim 100 \mathrm{~nm}$ to $\sim 2 \mu \mathrm{m}$. The components of Nye's tensor, from which dislocation density map can be calculated, are derived from local lattice curvatures according to their orientation variation with respect to the sample reference frame. The dislocation density distribution map overlaid with the reconstructed grain boundaries of the same region is represented in Fig. (2b). Interestingly, in a very small area two grains with two different dislocation architectures are observed. In the first one, shown by " 1 " in Fig. (2a), dislocations are almost uniformly distributed inside the grain, as shown in enlarged view in Fig. (2c). Quite the contrary, the 
second grain, shown by "2" letter in Fig. (2a), is almost depleted of dislocations, as shown in Fig. (2d). These, and other results, will be presented.

\section{References}

[1] E F Rauch, J Portillo, S Nicolopoulos, D Bultreys, S Rouvimov and P. Moeck, Zeitschrift für Kristallographie 225 (2010), p. 103.

[2] X Liu, N Nuhfer, A Rollett, S Sinha, S B Lee, J Carpenter, J LeDonne, A Darbal and K Barmak, Acta Materialia 64 (2013), p. 333.

[3] D. Viladot, M. Véron, M. Gemmi, F. Peiró, J. Portillo, S. Estradé, J. Mendoza, N. Llorca-Isern, S.

Nicolopoulos, Journal of microscopy 252 (2013), p. 23.

Figure 1. (a) Local orientation spread (LOS) plot of a simulated orientation database of $10^{*} 10$ cells with $0.6^{\circ}$ disorientation between the cells and $0.8^{\circ}$ noise in each pixel. (b) LOS plot of the filtered pattern.
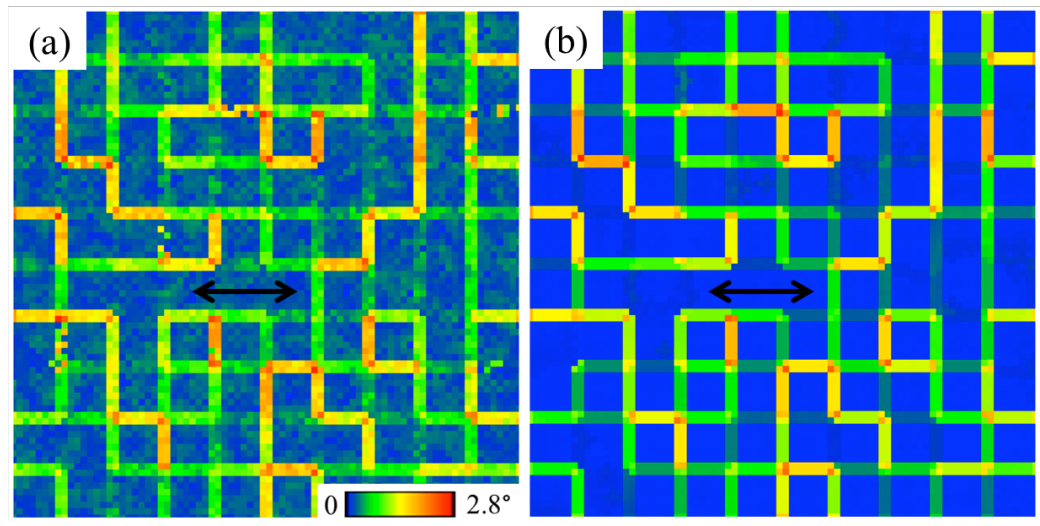

Figure 2. An example of the procedure proposed in this study (a) Index map (b) Dislocation density distribution map (c,d) Enlarged views of "1" and "2", respectively.
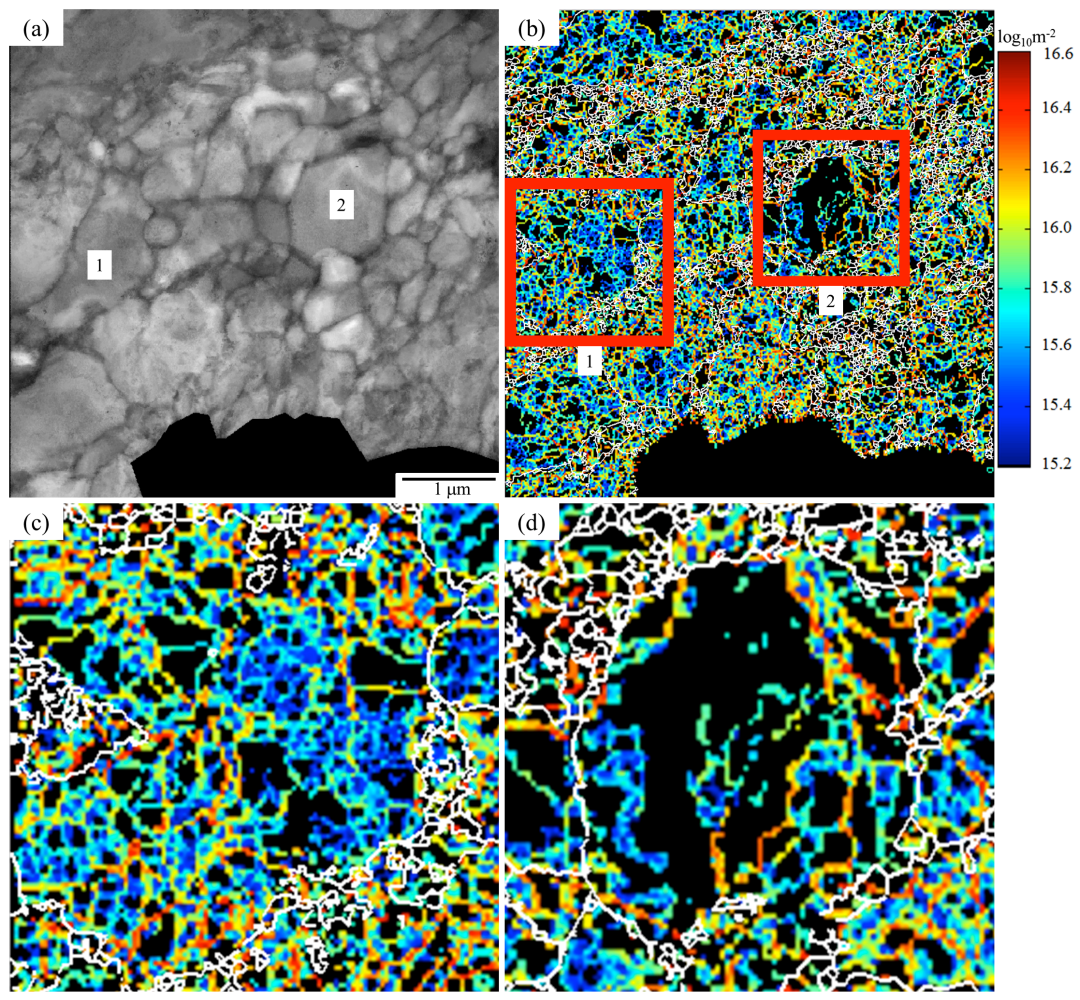